Notfall Rettungsmed $2023 \cdot 26: 54-56$

https://doi.org/10.1007/s10049-022-00987-0

Angenommen: 17. Januar 2022

Online publiziert: 9. Februar 2022

(c) Der/die Autor(en) 2022

\title{
Abrupt einsetzendes Erbrechen und Wesensveränderung
}

\author{
Martin Dombrowski ${ }^{1,2}$ (D) - Sarah Panahabadi ${ }^{3}$ \\ 'Abteilung für Anästhesie, Intensiv- und Notfallmedizin, Uniklinik Bonn, Bonn, Deutschland \\ ${ }^{2}$ Feuerwehr und Rettungsdienst, Bundesstadt Bonn, Bonn, Deutschland \\ ${ }^{3}$ Abteilung für Neuroradiologie, Uniklinik Bonn, Bonn, Deutschland
}

Die Alarmierung von Rettungswagen (RTW) und Notarzt erfolgte in den frühen Abendstunden bei vermutlich bewusstlosem Patienten.

\section{Befund}

Im Wohnzimmer saß der 83-jährige Patient auf einem Stuhl, gestützt von der Ehefrau, in der rechten Hand einen Eimer, in den er sich nahezu ohne Unterbrechung übergab.

Auffällig waren das fahle Hautkolorit mit ausgeprägter Kaltschweißigkeit, eine fragliche Fallneigung nach rechts, bei wiederholt krampfhaftem Griff an das Epigastrium.

Er wirkte hochgradig agitiert und antwortete nicht adäquat auf Fragen, die Pupillen zeigten sich beidseits stark verengt, der rechte Mundwinkel und das Augenlid schienen leicht zu hängen. Die Atmung war schnell, ohne pathologische Geräusche und suffizient. Eine Blutdruckmessung war aufgrund der Agitation sowie mehrlagiger Bekleidung zunächst nicht möglich, der Puls war jedoch gut tastbar und rhythmisch.

Wegen der räumlichen Enge sowie des blickdiagnostisch schwer kompromittierten Patienten erfolgte nach Anlage eines Zugangs der Transport in den RTW. Beim Aufrichten des Patienten zum Unterschieben des Tragetuchs zeigte sich eine Standunsicherheit.

Nach Gabe von $8 \mathrm{mg}$ Ondansetron und Beginn der Volumengabe erfolgte die ausführliche klinische Untersuchung.

Bei weiterhin ausgeprägter Übelkeit sowie Erbrechen von geringen Mengen unauffälligen Mageninhalts und Schleims konnte der Patient leicht dysarthrisch seinen Namen und das Geburtsdatum korrekt angeben. Arme und Beine wurden mit guter Kraft gezielt bewegt, die faziale Motorik war unauffällig. Die beidseitige Miosis zeigte sich nach wie vor. Fragen nach Schwindel oder Kopfschmerzen wurden nicht klar beantwortet.

$\mathrm{S}_{\mathrm{p}} \mathrm{O}_{2} 95 \%$, Blutdruck $145 / 80 \mathrm{~mm} \mathrm{Hg}$ Puls 100/Minute, am ehesten Sinusrhythmus (Artefakt-EKG bei schlechter Elektrodenhaftung), Temperatur $35,9^{\circ} \mathrm{C}$ und Blutzucker $123 \mathrm{mg} / \mathrm{dl}$.

Das Abdomen war weich, ohne lokalen Druckschmerz oder Abwehrspannung.

Kurze Zeit später zeigte die Pulsoxymetriekurve schnelle, regelmäßige Oszillationen bei unveränderter Vigilanz des Patienten (kontinuierliche EKG-Überwachung wegen der Kaltschweißigkeit unmöglich). Nach Aufkleben der Defi-Patches wurde eine regelmäßige Schmalkomplextachykardie von 140 bis 150 Schlägen pro Minute abgeleitet. Bei weiterhin gut tastbarem Puls und unveränderter Vigilanz wurde auf eine Therapie verzichtet. Die Tachykardie konvertierte nach knapp 2 min mit kurzzeitiger Pause in eine normofrequente Arrhythmie. In der Folge bestand ein fortwährender Wechsel zwischen regel-

\section{Infobox 1}

Symptome des Basilarisverschlusses.

(Übersetzt und modifiziert nach [3])

- Bewusstseinsstörung (Desorientiertheit, Vigilanzminderung, Koma)

- Störung von Atmung und Kreislauf

- Hypästhesien und Paresen (bis Tetraplegie)

- Standunsicherheit, Fallneigung, Nystagmus

- Okulomotoriusparese, Anisokorie

- Dysarthrie, Dysphagie

- Hemianopsie, kortikale Blindheit 

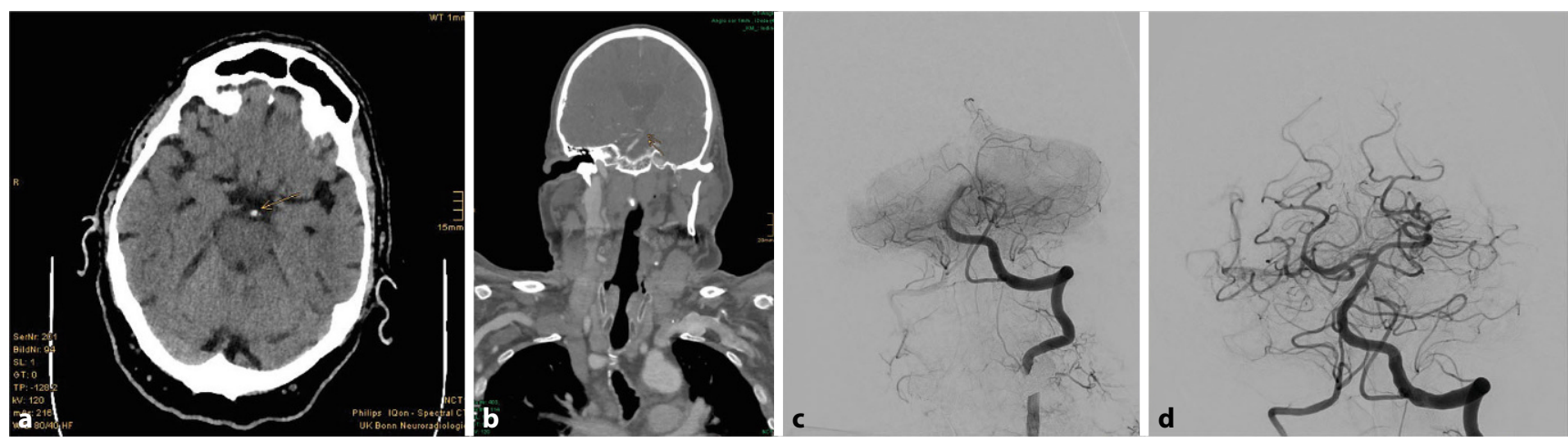

Abb. $1 \Delta$ a CCT nativ mit "dense artery sign" (gelber Pfeil). b CT-Angiographie mit Basilarisspitzenthrombose (gelbe Pfeile). c Angiographie vor Rekanalisierung. d Angiographie nach Rekanalisierung

mäßiger Schmalkomplextachykardie und normofrequenter Arrhythmie.

\section{Anamnese}

Über die Ehefrau war zu erfahren, dass sich ihr Mann, der den ganzen Tag völlig normal gewesen sei, alleine in der ersten Etage aufgehalten habe, als sie plötzlich ein Stöhnen vernommen habe. Sie habe ihn dann auf dem Stuhl sitzend und sich übergebend vorgefunden. Weil er nicht richtig ansprechbar gewesen sei, habe sie den Notarzt gerufen.

Auf die Mimik angesprochen, sagte sie, dass ihr Mann nicht anders aussehe als sonst. An Vorerkrankungen bestehe nur eine Hypertonie, weswegen er Ramipril und Metoprolol nehme.

\section{Diagnose}

Wegen des abrupt einsetzenden Erbrechens und der Agitation des nicht durchgehend adäquaten Patienten lag der Verdacht auf ein neurologisches Krankheitsbild nahe, auch wenn der Patient orientiert erschien und kein grob auffälliges sensomotorisches Defizit zu erheben war. Aufgrund der starken Kaltschweißigkeit und offenbar erstmalig aufgetretenen Rhythmusstörung erfolgte die telefonische Anmeldung in der Neurologie des nahegelegenen Uniklinikums anstelle der rein neurologischen Fachklinik.

Nach Ankunft in der Klinik wurde das EKG vom diensthabenden Kardiologen als Vorhofflimmern ohne Ischämieveränderungen und ohne Notwendigkeit zur sofortigen Intervention bewertet.
Nach Übergabe an den Dienstarzt der Neurologie erfolgte primär eine native CT des Kopfes, in der eine Blutung ausgeschlossen wurde. Allerdings ergab sich der Verdacht auf ein "hyperdense artery sign“ im Verlauf der Arteria (A.) basilaris (-Abb. 1a). In der nachfolgenden Angiographie (- Abb. 1b) wurde eine Basilarisspitzenthrombose festgestellt.

\section{Therapie und Verlauf}

Der Patient erhielt sofort eine systemische intravenöse Lyse mit $90 \mathrm{mg}$ rt-PA, angepasst an das Körpergewicht.

Außerdem wurde der verschlossene Gefäßabschnitt mittels Thrombektomie via Stent-Retriever eröffnet (- Abb. 1c, d). Die knapp 30-minütige Intervention gelang komplikationslos.

Postinterventionell klagte der Patient nur noch über Übelkeit, die unter der Gabe von Ondansetron rückläufig war. Neurologische Ausfallserscheinungen waren nicht nachweisbar. In der Verlaufsbildgebung war nur eine minimale Infarktdemarkation im betroffenen Stromgebiet zu erkennen.

Als ursächlich wurde eine Embolie kardialer Genese bei neu diagnostiziertem Vorhofflimmern angesehen, das in den folgenden Tagen weiter nachweisbar war. Es wurden eine orale Antikoagulation mit Apixaban und wegen rezidivierender blutdruckrelevanter Episoden einer Tachyarrhythmia absoluta die Aufdosierung von Digitalis begonnen.

Zwei Tage nach dem Ereignis konnte der beschwerdefreie Patient dem behandelnden Notarzt die Notfallsituation aus seiner Sicht schildern. Er habe ferngesehen, als er plötzlich einen Sehverlust erlitten habe.
Er habe sich elend gefühlt und andauernd erbrechen müssen. Es sei ihm nur möglich gewesen, auf Nachfrage seinen Namen und das Geburtsdatum zu nennen, die neu aufgetretene Blindheit habe er nicht artikulieren können. Das leichte rechtsseitige Hängen von Mundwinkel und Lid seien tatsächlich seit Jahren bekannt.

Wenige Tage später konnte der Patient in die ambulante Weiterbehandlung entlassen werden.

\section{Diskussion}

Gefäßverschlüsse der hinteren zerebromedullären Strombahn sind selten. Lediglich 20-25\% aller ischämischen Schlaganfälle betreffen dieses Gebiet. Verschlüsse der A. basilaris sind sogar nur in ca. $1-4 \%$ der Fälle zu finden [4]. Ursächlich sind nahezu zu gleichen Teilen Stenosen aufgrund einer Atherosklerose (26-36\%) sowie Embolien aus den Herzhöhlen oder großen arteriellen Gefäßen (30-35\%). Während atherosklerotische Veränderungen vor allem zu Verschlüssen am Übergang der Aa. vertebrales in die proximale $A$. basilaris führen, sind distale Basilarisverschlüsse wie im vorliegenden Fall typisch für Embolien $[3,6]$. Mit dem erstdiagnostizierten Vorhofflimmern konnte eine Quelle gefunden werden.

Die Symptomkonstellationen eines Basilarisverschlusses äußern sich in Abhängigkeit vom Ort des Verschlusses vielgestaltig ( $\mathbf{0}$ Infobox 1). Die Mortalität ist selbst unter Therapie mit $40-50 \%$ hoch [5], unbehandelt jedoch fatal, weswegen das Zeitfenster für mechanische Rekanalisationen mit bis $\mathrm{zu} 12 \mathrm{~h}$ angegeben wird [2]. 
Typisch für die Lokalisation des Gefäßverschlusses im vorliegenden Fall ist eine Symptomkonstellation, die als Basilarisspitzensyndrom beschrieben wird. Dominierend sind okuläre Symptome (Veränderungen der Okulo- sowie Pupillomotorik), kortikale Blindheit und Verhaltensauffälligkeiten (z. B. Agitation), die nicht selten als Delir fehlgedeutet werden. Grobe sensomotorische Defizite fehlen häufig [1].

Im Einsatz waren eine geringe Bewusstseinsstörung mit Agitation und Erbrechen sowie Fallneigung auffällig, außerdem die beidseitige Miosis bei unauffälliger Okulomotorik. Der Face-arm-speech-time-Test (FAST) war positiv bei Dysarthrie (jedoch nicht ausgeprägt), das Hängen von Mundwinkel und Lid war vorbekannt. Nicht erkannt wurde bei partieller Aphasie die kortikale Blindheit.

Eine Lebensmittelvergiftung oder eine Intoxikation mit Opioiden (stecknadelkopfgroße Pupillen/Erbrechen) war fremdanamnestisch nahezu auszuschließen. Die ausgeprägte Kaltschweißigkeit zusammen mit den neu aufgetretenen Herzrhythmusstörungen ließen eine relevante kardiale Beteiligung vermuten, weswegen der nahe Maximalversorger anstelle der rein neurologischen Fachklinik angefahren wurde.

Diese Entscheidung erwies sich als richtig, da der Patient ohne Zeitverlust kardiologisch evaluiert werden konnte und auch die Thrombektomie, bei 24-stündiger Thrombektomiebereitschaft, zügig, knapp $2 \mathrm{~h}$ nach Alarmierung des Notarztes, durchgeführt wurde.

\section{Fazit}

- Ischämien der hinteren zerebralen Strombahn können vielgestaltig auftreten. Grob auffällige sensomotorische Defizite fehlen.

- Das Zeitfenster für die mechanische Rekanalisation bei Basilarisverschlüssen beträgt bis zu $12 \mathrm{~h}$.

\section{Korrespondenzadresse}

\section{Dr. Martin Dombrowski}

Abteilung für Anästhesie, Intensiv- und

Notfallmedizin, Uniklinik Bonn

Venusberg-Campus 1, 53127 Bonn,

Deutschland

martin.dombrowski@ukbonn.de

Funding. Open Access funding enabled and organized by Projekt DEAL.

\section{Einhaltung ethischer Richtlinien}

Interessenkonflikt. M. Dombrowski und S. Panahabadi geben an, dass kein Interessenkonflikt besteht.

Für diesen Beitrag wurden von den Autoren keine Studien an Menschen oder Tieren durchgeführt. Für die aufgeführten Studien gelten die jeweils dort angegebenen ethischen Richtlinien. Für Bildmaterial oder anderweitige Angaben innerhalb des Manuskripts, über die Patienten zu identifizieren sind, liegt von ihnen und/oder ihren gesetzlichen Vertretern eine schriftliche Einwilligung vor.

Open Access. Dieser Artikel wird unter der Creative Commons Namensnennung 4.0 International Lizenz veröffentlicht, welche die Nutzung, Vervielfältigung, Bearbeitung, Verbreitung und Wiedergabe in jeglichem Medium und Format erlaubt, sofern Sie den/die ursprünglichen Autor(en) und die Quelle ordnungsgemäß nennen, einen Link zur Creative Commons Lizenz beifügen und angeben, ob Änderungen vorgenommen wurden.

Die in diesem Artikel enthaltenen Bilder und sonstiges Drittmaterial unterliegen ebenfalls der genannten Creative Commons Lizenz, sofern sich aus der Abbildungslegende nichts anderes ergibt. Sofern das betreffende Material nicht unter der genannten Creative Commons Lizenz steht und die betreffende Handlung nicht nach gesetzlichen Vorschriften erlaubt ist, ist für die oben aufgeführten Weiterverwendungen des Materials die Einwilligung des jeweiligen Rechteinhabers einzuholen.

Weitere Details zur Lizenz entnehmen Sie bitte der Lizenzinformation auf http://creativecommons.org/ licenses/by/4.0/deed.de.

\section{Literatur}

1. Caplan LR (1980) "Top of the basilar" syndrome. Neurology 30:72-79. https://doi.org/10.1212/ WNL.30.1.72

2. Lindsberg PJ, Pekkola J, Strbian D et al (2015) Time window for recanalization in basilar artery occlusion: speculative synthesis. Neurology 85:1806-1815. https://doi.org/10.1212/WNL. 0000000000002129

3. MattleHP, ArnoldM, Lindsberg PJetal (2011)Basilar artery occlusion. Lancet Neurol 10:1002-1014. https://doi.org/10.1016/S1474-4422(11)70229-0

4. Nagel S (2017) Schlaganfall bei akutem Verschluss der A. basilaris: Diagnostik und Therapie. Med Klin Intensivmed Notfmed 112:679-686. https://doi. org/10.1007/s00063-017-0347-0
5. Schonewille WJ, Wijman CAC, Michel P et al (2007) The basilar artery international cooperation study (BASICS). Int J Stroke 2:220-223. https://doi.org/ 10.1111/j.1747-4949.2007.00145.x

6. Schonewille WJ, Wijman CAC, Michel P et al (2009) Treatment and outcomes of acute basilar artery occlusion in the Basilar Artery International Cooperation Study (BASICS): a prospective registry study. Lancet Neurol 8:724-730. https://doi.org/ 10.1016/S1474-4422(09)70173-5 